\title{
"To do or not to do - that is the question". Transvascular needle aspiration during EBUS (EBUS-TVNA) with review of the literature
}

\begin{abstract}
Introduction: Large vessels are often encountered during endobronchial ultrasound (EBUS). Safety of traversing the vessels weighed against a more invasive procedure can be a dilemma.

Material and methods: We describe a case series of 8 patients who underwent transvascular needle aspiration during EBUS, to access a lesion in the absence of an alternate safe window. A 21 gauge EBUS needle was used to traverse either the main or a major branch of the pulmonary artery.

Results: Malignancy was suspected at ROSE in five cases. Granuloma and necrosis noted in 2 cases were confirmed as tuberculosis on culture. Diagnostic yield of EBUS-TVNA was $87.5 \%$ (7/8). No complications were noted in the immediate post-operative period as well as during 6 months of follow up.

Conclusion: EBUS-TVNA in carefully selected patients is a feasible alternative to more invasive procedures with excellent yield. Appropriate intraoperative, perioperative and postoperative monitoring and care must be available in the case of fatal bleeds.
\end{abstract}

Key words: EBUS, transvascular, TVNA, transvascular needle aspiration, transvascular biopsy

Adv Respir Med. 2021; 89: 386-391

\section{Introduction}

Invasive sampling of the mediastinal lymph nodes for lung cancer staging is recommended by American Thoracic Society (ATS), European Respiratory Society (ERS), and American College of Chest Physicians (ACCP) [1]. Minimal invasive procedure - endobronchial ultrasound (EBUS) [2] - guided needle biopsy is a well proven diagnostic tool for both malignant and nonmalignant diseases [3].

EBUS has, for the most part, made a greater portion of the mediastinum accessible to biopsies, especially when combined with EUS-B (transesophageal endobronchial ultrasound). Some stations in the mediastinum are inaccessible or, access to these locations is limited by the presence of one of the great vessels. These areas include stations 3a, 5 and 6 and elsewhere in the hilum when a branch of the pulmonary artery or more proximally, azygos, superior vena cava or brachiocephalic vessels, may be interposed between the airway and the pathology. These were once upon a time considered beyond the reaches of EBUS [4] because biopsy of these lesions needed traversing the great vessels. Life-threatening bleeding with EBUS-TBNA resulting probably from accidental pulmonary artery puncture have been reported [5, 6]. Multiple case series have now been published confirming relative safety of traversal of the great vessels in experienced hands [7-11].

\section{Material and methods}

We retrospectively analyzed patients who underwent endobronchial ultrasound with transvascular needle aspiration (EBUS-TVNA), during a 3-year period from January 2018 to December 2020. Procedural informed consent was obtained, and the study being a retrospective observation, was approved by the institutional ethics committee.

The protocol followed for the patients was not any different than for the other cases of 

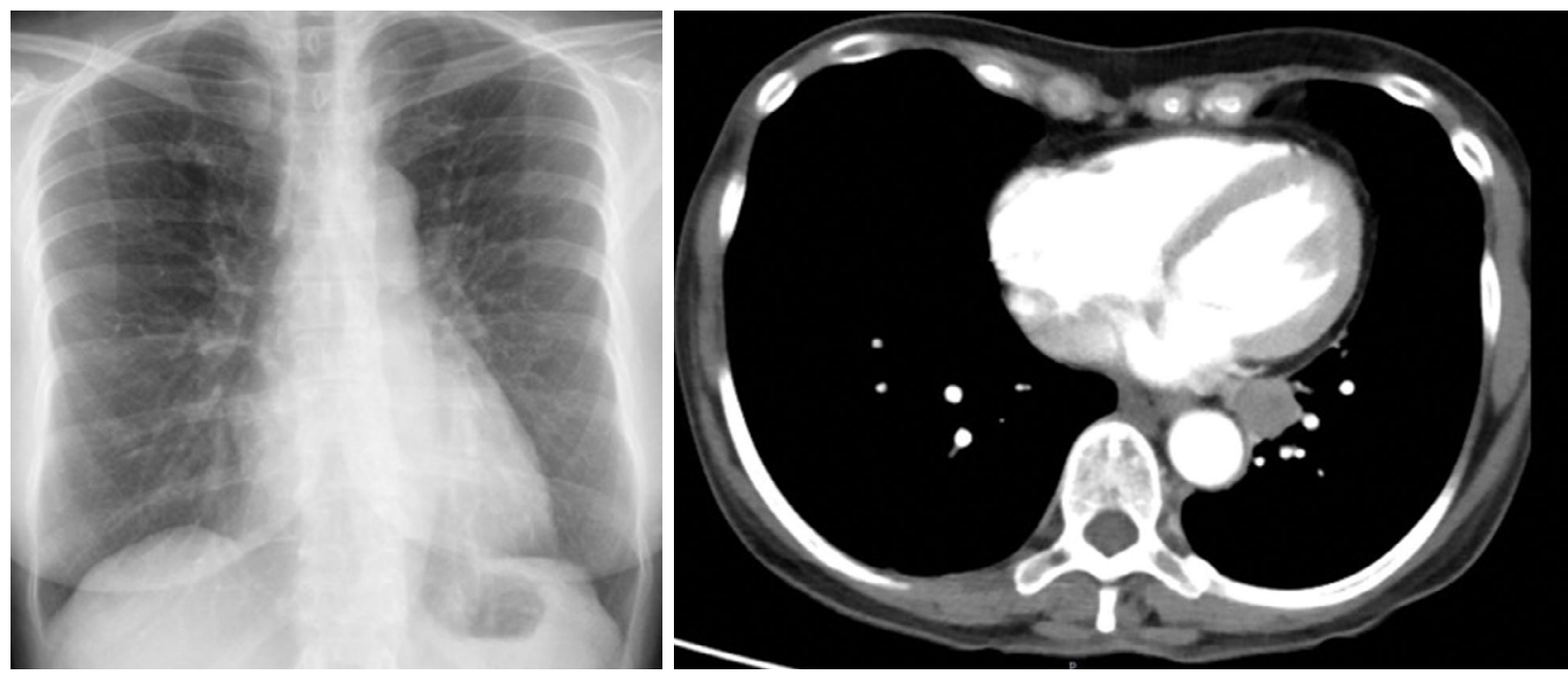

Figure 1. Retrocardiac lesion
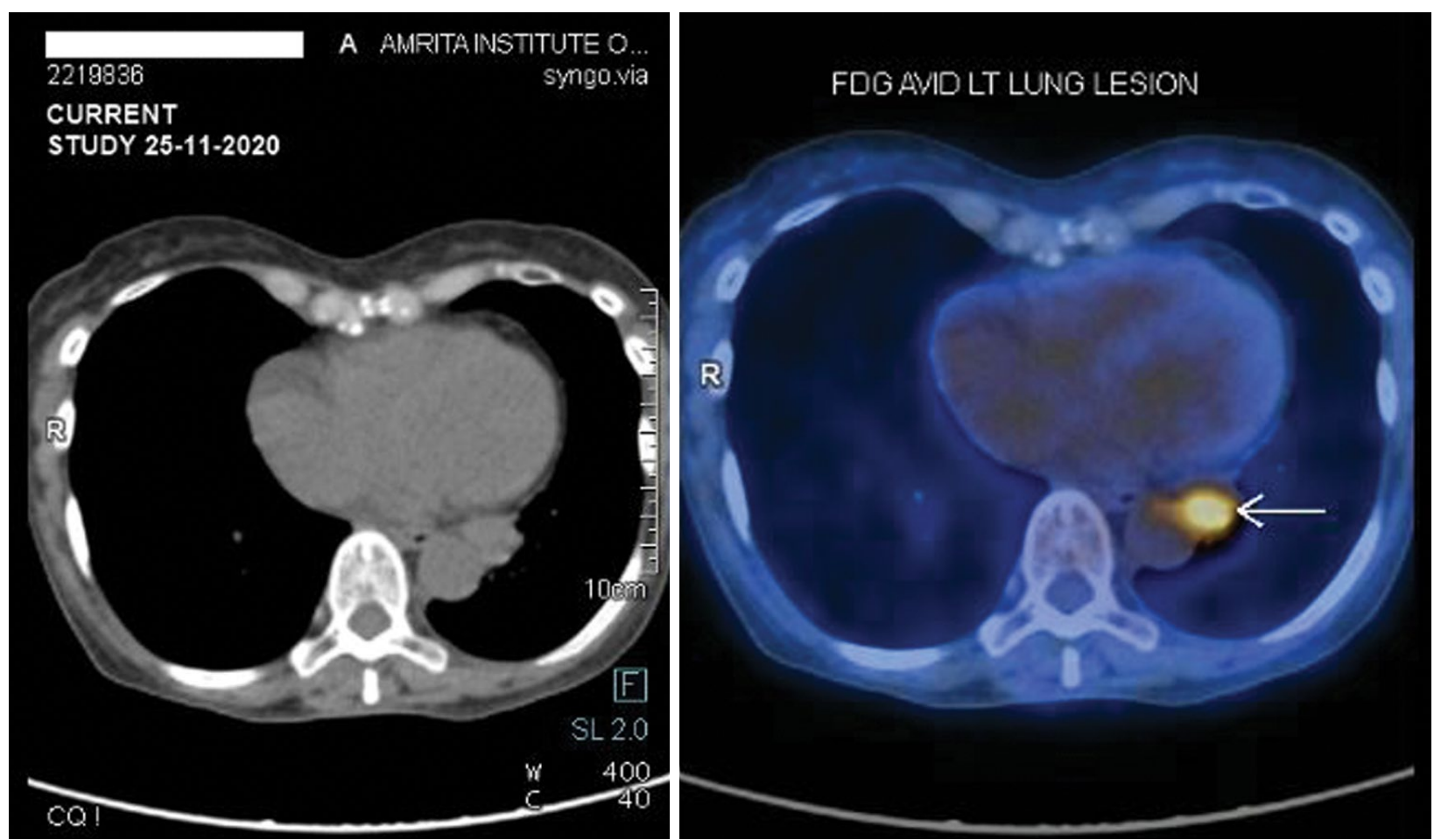

Figure 2. Fluorodeoxyglucose avid retrocardiac mass lesion

EBUS. Images available, including CT chest and/or PET-CT, were evaluated preoperatively (Figure 1 and 2). All patients posted for EBUS-TBNA at our unit are screened for cardiopulmonary and other systemic diseases and if necessary, further workup is done before the procedure. Basic blood work is always reviewed. Additionally, planning with the anesthesiology team included risks regarding major bleeding. Patients were type- and cross- matched. All the cases were consulted by experienced interventional pulmonologists. NSAIDs, anticoagulation and antiplatelet medications were discontinued. All the cases were planned as a day case with thoracic surgery backup.

A laryngeal airway was placed for general anesthesia and this allowed precision of biopsy and limited passes. Careful evaluation of the lesion and the vascular structures were done to find an appropriate window for biopsy. Every 
Table 1. Details of the cases who underwent transvascular needle aspiration during EBUS TVNA at Amrita Institute Of Medical Sciences, Aims Ponekkara, Kochi, India

\begin{tabular}{lcccccc}
\hline $\begin{array}{l}\text { Case no. \& } \\
\text { patient details }\end{array}$ & Indication & $\begin{array}{c}\text { Size of } \\
\text { lesion }\end{array}$ & $\begin{array}{c}\text { Vessel } \\
\text { traversed }\end{array}$ & $\begin{array}{c}\text { No of } \\
\text { passes }\end{array}$ & $\begin{array}{c}\text { EBUS rose } \\
\text { diagnosis }\end{array}$ & Final diagnosis \\
\hline $1.76 / \mathrm{M}$ & $\begin{array}{c}\text { Mass encasing } \\
\text { brachiocephalic } \\
\text { with right hilar mass }\end{array}$ & $26 \times 29$ & Interlobar PA & 2 & $\begin{array}{c}\text { Squamous cell } \\
\text { carcinoma }\end{array}$ & Esophageal cancer \\
2. 74/M & LLL mass & $23 \times 22$ & LMPA & 1 & Metastatic nsclca & Adenocarcinoma lung \\
$3.65 / \mathrm{M}$ & Station 5 lymph node & $25 \times 30$ & LMPA & 3 & Necrotic tissue & Tuberculosis \\
4. 53/F & LUL mass & $53 \times 39$ & LMPA & 3 & Adenocarcinoma & Adenocarcinoma lung \\
$5.55 / \mathrm{M}$ & Right hilar mass & $28 \times 27$ & Interlobar PA & 3 & Granuloma & Tuberculosis \\
$6.64 / \mathrm{F}$ & LLL mass/lymph node & $24 \times 17$ & LMPA & 3 & Nsclca & Adenocarcinoma lung \\
$7.47 / \mathrm{M}$ & Right hilar mass & $26 \times 29$ & Interlobar PA & 3 & $\begin{array}{c}\text { Suspicious } \\
\text { for malignancy }\end{array}$ & $\begin{array}{c}\text { Spindle Cell Neuroendocrine } \\
\text { neoplasm }\end{array}$ \\
$8.49 / \mathrm{F}$ & Left hilar mass encasing & $25 \times 28$ & LMPA & 3 & Nsclca & Adenocarcinoma \\
\hline
\end{tabular}

Interlobar PA — right main PA; NSCLCa — non small cell lung cancer; PA — pulmonary artery; LMPA — left main pulmonary artery; PA — pulmonary artery

attempt was made to avoid traversing the vasculature. Olympus scope BFUC180F was used for all the procedures. Twenty-one-gauge EBUS needle was used for the biopsy. Once the site of entry was finalized, the needle was advanced into the lesion traversing the vessel (Figure 3, 4). Ventilation was held during the puncture to avoid any shearing of the vessel wall. Once the needle was in the lesion, the stylet was removed, and the needle was moved back and forth 5 times with the application of suction. The sample was analyzed using rapid onsite evaluation (ROSE). Maximum of 3 needle passes were done for each of the lesions. After each pass, the airway was examined for bleeding and the vessel was checked for hematoma. Cold saline and epinephrine $(1: 10000)$ were used for hemostasis. Fogarty balloon catheter and a bronchial blocker was on standby for all the procedures.

\section{Results}

During the study period 2018-2020, 8 patients underwent EBUS-TVNA at our institution. The lesions were either at regular lymph node stations or had one of the vessels obstructing the needles direct access to the lesion.

Eight patients were identified out of which six had planned procedures done using general anesthesia, while in the other 2 cases, the decision was taken during the procedure done under procedural sedation. A transvascular approach was only adopted if a clear window to access the lesion for biopsy was absent.
Three cases involved station 5 where the left main pulmonary artery was traversed. Two cases needed traversing the common basal trunk of the left pulmonary artery. Three other cases needed traversing the interlobar pulmonary artery (right) for right hila masses. Six out of the eight cases were noted on ROSE to be suggestive of malignancy (75\%) and confirmed on final diagnosis. ROSE identified necrosis in 1 and granuloma in another subject, both of which were finally diagnosed as tuberculosis.

Four of the cases developed mild bleeding needing cold saline and epinephrine. No major bleeding was noted. Three needle passes were done to ensure adequacy of material for immunohistochemistry (IHC).

\section{Discussion}

Prior to the advent of EBUS, TBNA typically of station no. 7 (subcarinal) was a blind procedure done based on pre-procedure CT scans. Blood aspiration meant pulmonary artery puncture in which case, the needle was withdrawn and re-inserted at a different site if the airway was clean. EBUS-TBNA has prevented such accidental punctures. Fatal bleedings [5] have been reported in the literature, but EBUS-TBNA is for the most part a safe procedure [12]. Complications of hemomediastenum, hemothorax and pneumomediastinum after EBUS-TBNA [6] have been reported. Despite these concerns, multiple case series have documented relative safety of transvascular needle aspiration as summarized in Table 2 . 


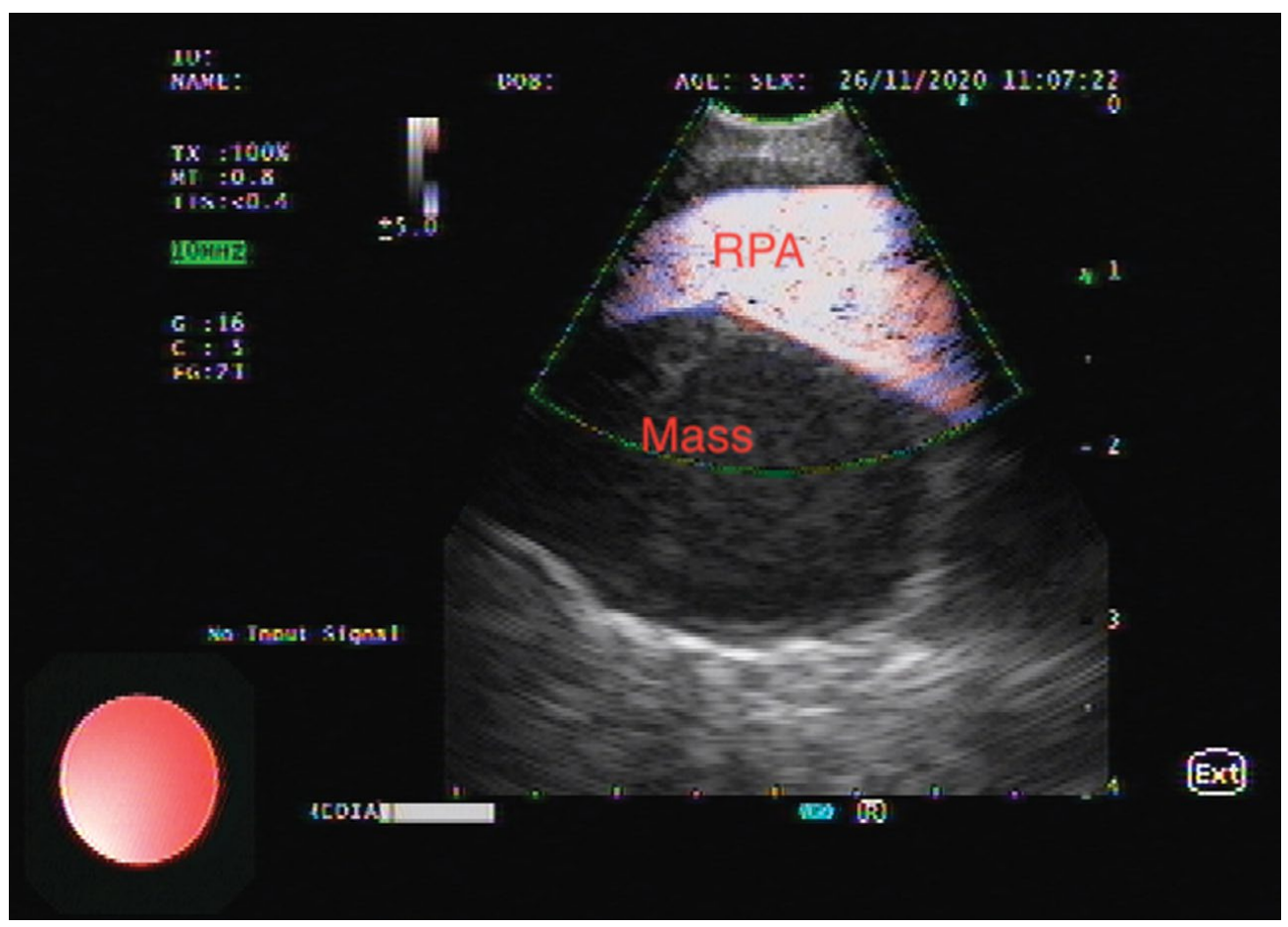

Figure 3. Lesion beyond the right main pulmonary artery with Doppler

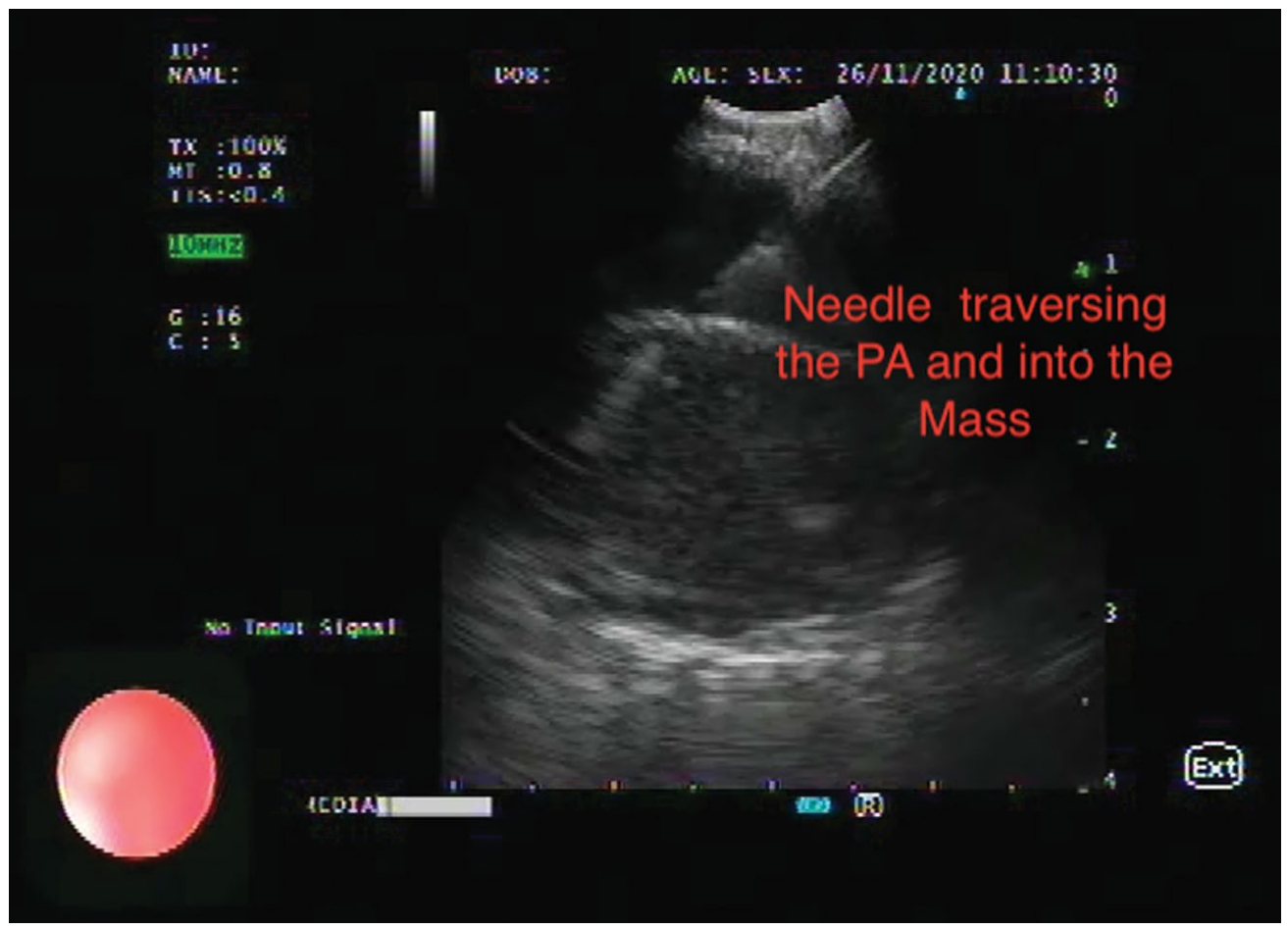

Figure 4. Transvascular needle aspiration during endobronchial ultrasound with transvascular needle aspiration. PA — pulmonary artery

Suprasternal puncture of the pulmonary artery has been recorded in the literature to be harmless dating back to the 1950's when the pulmonary arteries were inadvertently entered while aiming for the left atrium to do flow studies
[13]. Physiologically, the pulmonary artery system is a low-pressure high-capacitance structure. Anatomically, the pulmonary veins have a thin wall. The low-pressure system contributes to less bleeding with a small-bore needle (21-22 gauge) 
Table 2. Summary of cases series with endobronchial ultrasound with transvascular needle aspiration

\begin{tabular}{lccccc}
\hline Study & $\begin{array}{c}\text { Year of } \\
\text { publishing }\end{array}$ & $\begin{array}{c}\text { No. of } \\
\text { cases }\end{array}$ & Vessel traversed & Diagnostic yield [\%] & Complications \\
\hline Nuguru et al. [18] & 2017 & 12 & MPA/branch & 100 & None \\
$\begin{array}{l}\text { Naaman et al. [16] } \\
\text { (Abstract) }\end{array}$ & 2020 & 35 & $\begin{array}{c}\text { MPA/branch, } \\
\text { azygous, SVC, } \\
\text { brachiocephalic artery }\end{array}$ & $\begin{array}{c}\text { Unclear } \\
\text { but } \\
\text { diagnosed correctly }\end{array}$ & $\begin{array}{c}\text { 1 each of: respiratory failure, } \\
\text { atrial fibrillation-related tachy- } \\
\text { cardia, moderate bleeding }\end{array}$ \\
$\begin{array}{l}\text { Rachid MM [19] } \\
\text { (abstract) }\end{array}$ & 2018 & 24 & MPA/branch & 91.66 & None \\
Folch [9] & 2016 & 10 & MPA & 90 & None \\
Kazakov [8] & 2017 & 33 & MPA/branch, aorta & 73 & None \\
Panchabhai [11] & 2015 & 10 & MPA/branch & 90 & None \\
Mehta et al. [12] & 2018 & 10 & MPA/intra PA & 90 & None \\
Our study & 2020 & 8 & MPA/branch & 100 & None \\
Boujaoude et al. [10] & 2013 & 2 & Branch MPA & 100 & None \\
Cetinkaya et al. [20] & 2018 & 4 & MPA/branch & 100 & None \\
\hline
\end{tabular}

LMPA — main pulmonary artery; PA — pulmonary artery

biopsy, but the thin wall makes the risk of tear higher. Longer needle dwell time in the vessel and respiratory excursions increase the risk of vessel injury. The pulmonary artery is in close proximity to a significant portion of the airway and need for EBUS-TVNA is not unusual.

Boujaoude et al. [9] reported safety of puncturing the main pulmonary artery in 2013 in 2 cases and since then most of the vessels seen during EBUS $[7,14]$ have been traversed. Intrapulmonary artery lesions have also been sampled with good yield and safety profile [11, 15].

General anesthesia is used in most of the case series except Folch et al. [18] and Kazakov et al. [7]. Just as in our series, they also noted that anesthesia did not influence the complications. In a large series of 35 patients, Naaman et al. [14] (published as an abstract) noted reversible mild to moderate complication in only 3 patients, and all of them recovered fairly well without any major sequalae. Similarly, puncturing other vessels, e.g. azygous, SVC and brachiocephalic artery did not change the risk [14]. Our study involved puncturing the main pulmonary artery at different sites, and we did not notice any increased complication rate just as noted by Panchabhai et al. [10]. Kazakov et al. [7] in their series traversed the aorta as well and did not notice any increase in complications.

The average diagnostic yield for the EBUS-TVNA in various case series seems to be well over $80 \%$. We found a diagnosis in $87 \%$ in our study, though in 2 of the cases, the definitive diagnosis was not confirmed immediately but was ultimately established as tuberculosis. ROSE had identified granuloma and necrosis. As with the other case series, overall final diagnosis of malignancy was very high in our series as well, i.e., at 75\% (6/8).

Findings from our study add to the growing literature of safety and excellent diagnostic yield of EBUS-TVNA in selective cases.

Our case series has several limitations. The sample size was small, and this was a retrospective chart review which carries its drawbacks. The small number is probably related to the relative low frequency of these types of cases seen in clinical practice, which was also noted in many other series in the literature. Ours was also a single-center experience. Being retrospective in nature, subtle postoperative complications could have been missed. We had an excellent diagnostic yield but did not have another procedure to compare with. Overinterpreting of our results, both diagnostic yield and the absence of complications, would be unwise and we would strongly advice against that.

The EBUS-TVNA should only be attempted as a last resort after weighing all options and is not a replacement for the more invasive procedure like mediastinoscopy. Adequate preoperative, intraoperative and perioperative planning, monitoring and taking precautions like cardiothoracic surgery on standby, are the keys to doing a successful procedure. The opinion as mentioned in the various case series is one of caution. Preferably, this procedure should be attempted in a high-volume center and only by skilled interventional pulmonologists. We strong- 
ly recommend choosing the patient carefully and always attempting to avoid vessel, even though EBUS-TVNA appears to be a relatively safe procedure. While general anesthesia seems to be the common consensus for this high-risk procedure, cases done under conscious sedation seem to have similar results. But we would still advice taking a safer approach to this procedure.

\section{Conclusion}

In conclusion, using transvascular approach expands the stations and areas in the mediastinum amenable to biopsy during EBUS. EBUS-TVNA appears to be a relatively safe procedure in skilled hands, but safety precautions with strict adherence to protocols and patient selection are advised.

\section{Conflict of interest}

None declared.

\section{References:}

1. Detterbeck FC, Postmus PE, Tanoue LT. The stage classification of lung cancer: Diagnosis and management of lung cancer, 3rd ed: American College of Chest Physicians evidence-based clinical practice guidelines. Chest. 2013; 143(5 Suppl): e191S-e210S, doi: 10.1378/chest.12-2354, indexed in Pubmed: 23649438.

2. Detterbeck FC, Boffa DJ, Kim AW, et al. The eighth edition lung cancer stage classification. Chest. 2017; 151(1): 193-203, doi: 10.1016/j.chest.2016.10.010, indexed in Pubmed: 27780786.

3. Ortakoylu MG, Iliaz S, Bahadir A, et al. Diagnostic value of endobronchial ultrasound-guided transbronchial needle aspiration in various lung diseases. J Bras Pneumol. 2015; 41(5): 410-414, doi: 10.1590/S1806-37132015000004493, indexed in Pubmed: 26578131.

4. Jalil BA, Yasufuku K, Khan AM. Uses, limitations, and complications of endobronchial ultrasound. Proc (Bayl Univ Med Cent). 2015; 28(3): 325-330, doi: 10.1080/08998280.2015.11929263, indexed in Pubmed: 26130878.
5. Miller DR, Mydin HH, Marshall ADL, et al. Fatal haemorrhage following endobronchial ultrasound-transbronchial needle aspiration: an unfortunate first. QJM. 2013; 106(3): 295-296, doi: 10.1093/qjmed/hct005, indexed in Pubmed: 23359720.

6. King C, Rendo M, Kruse B, et al. Near fatal hemomediastinum with hemopneumothorax following endobronchial ultrasound-guided biopsy. Chest. 2019; 156(4): A1507-A1508, doi: 10.1016/j.chest.2019.08.1334.

7. Kazakov J, Hegde P, Tahiri M, et al. Endobronchial and endoscopic ultrasound-guided transvascular biopsy of mediastinal, hilar, and lung lesions. Ann Thorac Surg. 2017; 103(3): 951-955, doi: 10.1016/j.athoracsur.2016.08.111, indexed in Pubmed: 27865476.

8. Folch E, Santacruz JF, Fernandez-Bussy S, et al. The feasibility of EBUS-guided TBNA through the pulmonary artery in highly selected patients. J Bronchology Interv Pulmonol. 2016; 23(1): 7-13, doi: 10.1097/LBR.0000000000000245, indexed in Pubmed: 26705006.

9. Boujaoude Z, Pratter M, Abouzgheib W. Transpulmonary artery needle aspiration of hilar masses with endobronchial ultrasound: a necessary evil. J Bronchology Interv Pulmonol. 2013; 20(4): 349-351, doi: 10.1097/LBR.0000000000000011, indexed in Pubmed: 24162122.

10. Panchabhai TS, Machuzak MS, Sethi S, et al. Endobronchial ultrasound-guided transvascular needle aspiration: A single-center experience. J Bronchology Interv Pulmonol. 2015; 22(4): 306-311, doi: 10.1097/LBR.0000000000000227, indexed in Pubmed: 26492603.

11. Mehta RM, Biraris PR, Pattabhiraman V, et al. Defining expanded areas in EBUS sampling: EBUS guided trans- and intra-pulmonary artery needle aspiration, with review of transvascular EBUS. Clin Respir J. 2018; 12(5): 1958-1963, doi: 10.1111/ crj.12764, indexed in Pubmed: 29330908.

12. Eapen GA, Shah AM, Lei X, et al. American College of Chest Physicians Quality Improvement Registry, Education, and Evaluation (AQuIRE) Participants. Complications, consequences, and practice patterns of endobronchial ultrasound-guided transbronchial needle aspiration: Results of the AQuIRE registry. Chest. 2013; 143(4): 1044-1053, doi: 10.1378/ chest.12-0350, indexed in Pubmed: 23117878.

13. Radner S. Suprasternal puncture of the pulmonary artery. Acta Med Scand. 1954; 148(2): 161-164, doi: 10.1111/j.09546820.1954.tb01708.x, indexed in Pubmed: 13157932.

14. Naaman R, Sheikh A, Diab K. The feasibility of transvascular endobronchial ultrasound-guided transbronchial needle aspiration: a single tertiary-care center experience with 35 patients. Chest. 2020; 158(4): A1953, doi: 10.1016/j.chest.2020.08.1690.

15. Harris K, Modi K, Kumar A, et al. Endobronchial ultrasound-guided transbronchial needle aspiration of pulmonary artery tumors: A systematic review (with video). Endosc Ultrasound. 2015; 4(3): 191-197, doi: 10.4103/2303-9027.162996, indexed in Pubmed: 26374576. 